miderine/sciences 1995; $11: 1629$

\title{
Claude Vézina (1926-1995)
}

\author{
Michel Bergeron
}

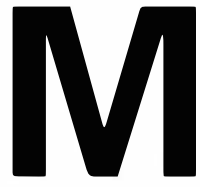

édecine/sciences vient de perdre un de ses plus fidèles soutiens. Claude Vézina a fait partie en 1983 du comité scientifique québécois dont les actions, jumelées à celles d'un comité français qu'animait Jean Hamburger, devaient aboutir à la création de médecine/sciences. Sa réputation de scientifique et son expérience d'administrateur en firent le candidat idéal pour présider le Conseil d'administration de la jeune Société de la Revue médecine/sciences, créée au Qućbec pour lancer la nouvelle revue franco-québécoise et en assurer l'organisation financière.

Agronome et microbiologiste de formation, diplômé de l'Institut Agronomique d'Oka au Québec, il obtint son doctorat $(\mathrm{PhD})$ de l'Université de Wisconsin et fut l'un des premiers à s'engager dans la génétique bactérienne. Rapidement recruté par les laboratoires Ayerst de Montréal, il dut décliner une offre de chercheur postdoctoral chez André Lwoff et François Jacob. Alors commence pour Claude Vézina une longue et fructueuse carrière en recherche industrielle qui l'amena rapidement à la direction du Département de microbiologie chez Ayerst.

L'équipe que dirigeait Claude Vézina décrivit de nouveaux procédés de bioconversion par les spores des champignons et des streptomycètes ainsi que de nouvelles méthodes de préparation des œstrogènes et de production par fermentation. Il synthétisa une centaine de nouveaux stéroïdes et de nouveaux antibiotiques dont la rapamycine est le plus connu (médecine) sciences 1995; 10 (suppl 1): 3-4). Plus de 25 brevets furent déposés au Canada, aux USA et dans la majorité des pays industrialisés : ils portent sur de nouveaux antibiotiques, stéroïdes et procédés de fermentation. Claude Vézina étonnait par son érudition, ses connaissances encyclopédiques, son imagination et son grand jugement, toutes qualités qui l'ont conduit, au sein de diverses sociétés ou institutions, à des postes de direction, dont le dernier comme directeur de projets spéciaux d'une des entreprises les plus dynamiques de Montréal, BioChem Thérapeutique.
Claude Vézina fut aussi un citoyen exemplaire: il fut notamment président de la Commission scolaire d'Oka et fut choisi comme premier président du Conseil des commissaires de la Commission scolaire de sa région. Au cours des 10 premières années de médecine/sciences, Claude Vézina présida aux destinées du Conseil d'administration de la Société de la Revue médecine/sciences qui put profiter de sa vaste expérience d'administrateur et de sa connaissance des milieux industriels, universitaires et financiers.

médecine/sciences perd un de ses plus fidèles lecteurs, un des fervents pionniers de cette aventure. Nous perdons un grand scientifique et un ami

\section{Michel Bergeron}

Département de physiologie, université de Montréal, CP 6128, Montréal, Québec, H3C 3J7 Canada. 